\title{
THE INTERNET-BASED TELEOPERATION: MOTION AND FORCE PREDICTIONS USING THE PARTICLE FILTER METHOD
}

\author{
Jae-young Lee \\ School of Engineering Science \\ Simon Fraser University \\ Burnaby, BC, Canada \\ jla155@sfu.ca
}

\author{
Shahram Payandeh \\ School of Engineering Science \\ Simon Fraser University \\ Burnaby, BC, Canada \\ shahram@cs.sfu.ca
}

\author{
Ljiljana Trajković \\ School of Engineering Science \\ Simon Fraser University \\ Burnaby, BC, Canada \\ ljilja@cs.sfu.ca
}

\section{ABSTRACT}

In this paper, we present motion and force predictions in Internet-based teleoperation systems using the particle filter method. The particle filter, also known as the sequential Monte Carlo (SMC) method, is a probabilistic prediction or estimation technique within a sequential Bayesian framework: Data at a current time step are predicted or estimated by recursively generating probability distribution based on previous observations and input states. In this paper, we first formulate the particle filter method using a prediction-based approach. Motion and force data flows, which may be impaired by the Internet delay, are formulated within a sequential Bayesian framework. The true motion and force data are then predicted by employing the prediction-based particle filter method using the impaired observations and previous input states. We performed experiments using a haptic device that interacts with a mechanics-based virtual 3D graphical environment. The haptic device is used as a master controller that provides positioning inputs to a 4-degree of freedom (4-DoF) virtual robotic manipulator while receiving feedback force through interactions with the virtual environment. We simulate the Internet delay with variations typically observed in a user datagram protocol (UDP) transmission between the master controller and the virtual teleoperated robot. In this experimental scenario, the particle filter method is implemented for both motion and force data that experience the Internet delay. The proposed method is compared with the conventional Kalman filter. Experimental results indicate that in nonlinear and non-Gaussian environments the prediction-based particle filter has distinct advantage over other methods.

\section{INTRODUCTION}

An Internet-based teleoperation system is an interactive application where though a master device, a human operator transmits motion data while simultaneously receiving reflecting force data from a slave robot controller. Unlike other Internet applications that mainly focus on the reliable data transmission, interactive applications are highly delay-sensitive. The Internet delay, which is unknown and varies over time according to network conditions, may cause instability of an overall teleoperation system. Furthermore, the transmitted motion and force data are often impaired by significant delay and delay jitter during the Internet transmission [1].

Various approaches have been suggested in order to solve the time delay issue of Internet-based teleoperation systems. In the area of control systems, the wave variables transformation and its extensions have focused on the stability of overall teleoperation systems in the presence of constant delay [2], [3], 
[4]. In the area of Internet transport protocols, several proposals have been suggested based on modifications to transport control protocol (TCP) and user datagram protocol (UDP) to enable faster transmissions of data packets [5], [6]. In the area of signal processing, prediction-based methods that perform motion and force predictions have been proposed [7], [8]. The Kalman filter method, which provides a recursive solution to the linear prediction and estimation, was proposed as a prediction-based approach [9]. These methods have been used to compensate for the transmitted motion and force data that are impaired by variations of the Internet delay.

Motion and force data are often difficult to predict in systems with nonlinear and non-Gaussian characteristics. For example, fine hand motion commands from a master controller may be highly nonlinear and the traditional Kalman filter may fail to provide their accurate prediction. Force data may be even more difficult to predict since the data need to be sent at relatively high frequencies to guarantee realistic force without discontinuity and to avoid closed-loop instability of an overall teleoperation system. Furthermore, the motion and force data may contain a non-Gaussian noise such as an impulse noise during the transmission, which leads to further challenges in prediction.

The particle filter method, also known as the bootstrap filter or the Condensation, is a sequential Monte Carlo (SMC) method that provides a sub-optimal solution in recursive Bayesian approaches [10], [11]. Due to its robust prediction and estimation performance in nonlinear and non-Gaussian environments, the particle filter method has been widely applied in the areas of communications, image and speech signal processing, control systems, and robotics [12], [13]. Since the particle filter method can be applied to any signal using a discrete time state-space formulation, it has been applied to nonlinear motion and force data flows in an Internet-based teleoperation system [14].

In this paper, we employ the particle filter method to predict motion and force data that may be nonlinear and nonGaussian in addition to being subject to the Internet delay. We first introduce the prediction-based particle filter method applied to motion and force data flows using a discrete time state-space formulation. We describe an experimental study based on the implemented particle filter method [14]. In this paper, the proposed method is verified in both nonlinear and non-Gaussian environments. We also present the comparison of prediction performance between the proposed particle filter and the conventional Kalman filter methods. The stability issue of an overall teleoperation system in the presence of the Internet delay is discussed.

\section{MOTION AND FORCE PREDICTIONS IN INTERNET- BASED TELEOPERATION SYSTEMS}

Motion data generated from a master controller are transmitted to a slave controller through the Internet. Based on the design of the slave controller, reflected force data are generated by any contact with an object or surrounding environment and they are fed into the master controller through the Internet. A simple illustration of motion and force data flows in an Internet-based teleoperation system is shown in Figure 1. The motion and force data are represented in discrete time statespace formulations, which are configured in a recursive Bayesian framework. Since the motion and force data experience variations of the Internet delay, the true data may be impaired and stability of an overall teleoperation system may not be maintained. In order to compensate for such Internet delay, we employ the prediction-based particle filter method for the motion and force data flows.

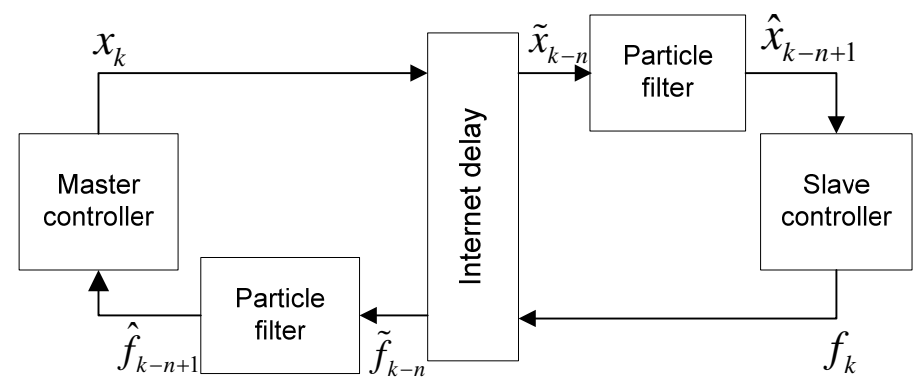

Figure 1. State-space formulations of motion and force data flows in an Internet-based teleoperation system. The proposed prediction approach employs the particle filter method.

\section{Motion Prediction}

The motion data flow in an Internet-based teleoperation system, which consists of positions samples over time, is represented as a discrete time state-space formulation as shown in Figure 1. In a single DoF teleoperation system, the true position $x_{k}$ at time $k$ is transmitted through the Internet and it is delayed by $n$ time steps. The impaired observation received at the slave controller can be expressed as $\tilde{x}_{k-n}$. The discrete time nonlinear motion data flow in a state-space formulation may be expressed as:

$$
\left\{\begin{array}{l}
x_{k}=g_{k}\left(x_{k}, u_{k}\right) \\
\tilde{x}_{k-n}=h_{k-n}\left(x_{k-n}, v_{k-n}\right)
\end{array},\right.
$$

where $x_{k}$ and $\tilde{x}_{k-n}$ are the system state at time $k$, and the system observation at time $k-n, g_{k}$ and $h_{k-n}$ are nonlinear state and observation transition functions, and $u_{k}$ and $v_{k-n}$ are 
state and observation noise sequences, which may be nonGaussian. In a state-space formulation, the prediction of the true position at time $k-n+1$ is calculated based on the current state $x_{k-n}$ and available observation $\tilde{x}_{1: k-n}$. In a recursive Bayesian approach, the optimal predictor of the true state at time $k-n+1$ is expressed by the conditional mean:

$$
\hat{x}_{k-n+1 \mid k-n}=\int x_{k-n+1} p\left(x_{k-n+1} \mid \tilde{x}_{1: k-n}\right) d x_{k-n},
$$

where $\hat{x}_{k-n+1 \mid k-n}$ denotes the prediction of the state $x_{k-n+1}$ given available observations $\tilde{x}_{1: k-n}$. The posterior density in (2) is evaluated recursively solving two density functions [9]:

$$
\begin{gathered}
p\left(x_{k-n+1} \mid \tilde{x}_{1: k-n}\right)=\int p\left(x_{k-n+1} \mid x_{k-n}\right) p\left(x_{k-n} \mid \tilde{x}_{1: k-n}\right) d x_{k-n} \\
p\left(x_{k-n} \mid \tilde{x}_{1: k-n}\right)=\frac{p\left(\tilde{x}_{k-n} \mid x_{k-n}\right) p\left(x_{k-n} \mid \tilde{x}_{1: k-n-1}\right)}{p\left(\tilde{x}_{k-n} \mid \tilde{x}_{1: k-n-1}\right)}
\end{gathered}
$$

Equations (3) and (4) provide the prediction and update procedures for the optimal solution, respectively. They are not computationally tractable due to their integral forms. Hence, the particle filter method is used to approximate the posterior density as a suboptimal solution. Based on the prediction-based particle filter method, (3) is approximated as [15], [16]:

$$
p\left(x_{k-n+1} \mid \tilde{x}_{1: k-n}\right) \approx \sum_{i=1}^{N_{s}} w_{k-n+1}^{i} \delta\left(x_{k-n+1}-x_{k-n+1}^{i}\right)
$$

where $N_{S}$ is the number of particles, $\delta(\cdot)$ is the Dirac delta function, and $w_{k-n+1}^{i}$ is the importance weight computed as:

$$
w_{k-n+1}^{i} \propto w_{k-n}^{i} \frac{p\left(\tilde{x}_{k-n+1} \mid x_{k-n+1}^{i}\right) p\left(x_{k-n+1}^{i} \mid x_{k-n}^{i}\right)}{q\left(x_{k-n+1}^{i} \mid x_{k-n}^{i}, \tilde{x}_{k-n+1}\right)} .
$$

In order to minimize the variance of importance weights, the importance density $q(\cdot)$ may be chosen to be equal to the prior density such that:

$$
q\left(x_{k-n+1} \mid x_{k-n}^{i}, \tilde{x}_{k-n+1}\right)=p\left(x_{k-n+1} \mid x_{k-n}^{i}\right) .
$$

Hence, the importance weight is simplified as:

$$
w_{k-n+1}^{i} \propto w_{k-n}^{i} p\left(\tilde{x}_{k-n} \mid x_{k-n}^{i}\right) .
$$

In this paper, we consider the motion data flow in nonlinear and non-Gaussian environments. Hence, we assume the state noise $u_{k}$ to have a Gamma distribution, which is typically observed in an impulse noise [17]. The non-Gaussian noise can be modeled using the Gamma density function:

$$
p\left(u_{k}\right)=\frac{\beta^{\alpha}}{(\alpha-1) !} u_{k}^{\alpha-1} e^{-\beta u_{k}} \text { for } u_{k} \geq 0 \text {. }
$$

The mean and variance of the Gamma density function (9) are $\alpha / \beta$ and $\alpha / \beta^{2}$, respectively. Note that the Gamma density function applies to the state noise $u_{k}$ and does not apply to the observation noise $v_{k}$. Given these noise assumptions, the importance weight (8) is further simplified as:

$$
w_{k-n+1}^{i}=e^{-\frac{1}{2 \sigma^{2}}\left(\tilde{x}_{k-n}-x_{k-n}^{i}\right)}
$$

Equation (10) gives the importance weight of the $i$-th particle at time $k-n+1$ and needs to be normalized so that:

$$
\sum_{i=1}^{N_{S}} w_{k-n+1}^{i}=1
$$

The resampling step should be performed to regenerate the predicted samples based on measurements of the importance weights. When cumulative distribution functions (CDF) of the normalized weights (11) are constructed, each element of the CDF is compared with a uniformly distributed function in order to determine whether the weights are high or low. Based on this comparison, particles with low weights are eliminated and particles with high weights are used to predict the true states. Therefore, a new set of states $x_{k-n+1}$ is determined and this procedure is repeated for the next time step. Each step of the particle filter method for the motion data prediction is described in Table 1.

Table 1. Particle Filter Method for Motion Prediction.

\section{Step 1. Initialization}

Randomly choose the initial state and define initial parameters

Step 2. Prediction

$$
\text { Evaluate } x_{k-n+1}^{i} \sim p\left(x_{k-n+1} \mid x_{k-n}^{i}\right), 1 \leq i \leq N_{S}
$$

Step3. Update

Evaluate importance weights (8) and normalize.

\section{Step 4. Resampling}

Multiply/suppress samples with high/low importance weights

Step 5. Iteration

Increase time step and go to Step 2. 


\section{Force Prediction}

Force data, generated by any contact with an object and fed into the master controller, may be predicted by a similar approach. As shown in Figure 1, the transmitted force data over the Internet may also be formulated within a nonlinear and nonGaussian state-space framework. The true force data are then predicted by the prediction-based particle filter method given available observations.

For a single DoF teleoperation system, let $f_{k}$ be the true force data generated by the slave controller at time $k$. The force data transmitted to the master controller through the Internet is delayed by $n$ time steps. This is the impaired observation that may be expressed as $\tilde{f}_{k-n}$. Similar to the representation of the motion prediction (2), the prediction of the true force $f_{k-n+1}$ is calculated based on the current state $f_{k-n}$ and available observations $\tilde{f}_{1: k-n}$. Hence, in a recursive Bayesian approach, the optimal predictor of the true force at time $k-n+1$ is given as:

$$
\hat{f}_{k-n+1 \mid k-n}=\int f_{k-n+1} p\left(f_{k-n+1} \mid \tilde{f}_{1: k-n}\right) d f_{k-n}
$$

Using the prediction-based particle filter method, the predicted force $\hat{f}_{k-n+1 \mid k-n}$ is calculated by approximating the posterior density function $p\left(f_{k-n+1} \mid \tilde{f}_{1: k-n}\right)$ by using (5)-(8).

Similar to the motion prediction case, the force prediction is performed by computing each step in Table 1. After the initialization step, which randomly selects an initial state of motion data, the prediction step is performed to obtain samples $f_{k-n+1}^{i}$ from the prior density $p\left(f_{k-n+1} \mid f_{k-n}^{i}\right)$, where $1 \leq i \leq N_{s}$. In the update step, the new state $f_{k-n+1}$ is assigned by using importance weights. In the force prediction case, we assume that the non-Gaussian state noise is modeled using the Gamma density function (9). Then, after normalizing the computed importance weights, the resampling step is performed in order to regenerate a new set of states $f_{k-n+1}$ based on high weighted samples.

\section{EXPERIMENTS}

In order to evaluate the proposed particle filter method for Internet-based teleoperation systems, we performed experiments using the PHANTOM Desktop haptic device as a master manipulator. The experimental setup consisting of the haptic device and the virtual robot based on a Selective Compliance
Assembly Robot Arm (SCARA) manipulator configuration is shown in Figure 2. Based on movements from the master controller, contact force data that feed into the master controller are generated when the tip of the SCARA manipulator collides with objects in the graphical scene. In this experiment, positions of the 4-DoF virtual SCARA robot are kinematically mapped to the master controller, which is able to manipulate 4-DoF. In conjunction with the mechanics-based model of the 3D graphical environment, the haptic device provides positioning inputs to the slave controller while receiving feedback force through interactions with the virtual environment.

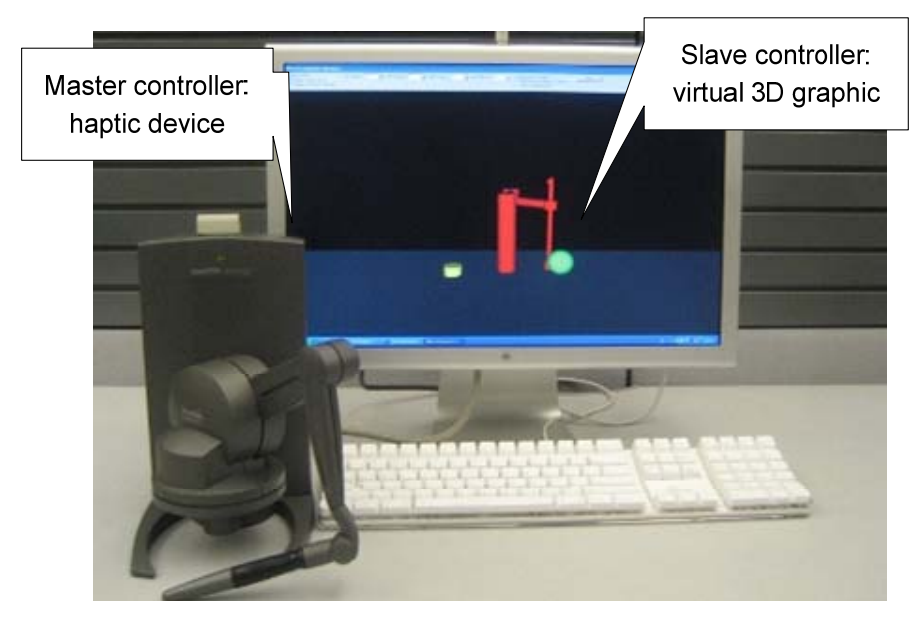

Figure 2. Experimental scenario: The PHANTOM Desktop haptic device and virtual 3D graphical representation are used for master and slave controllers, respectively.

TCP and UDP are two widely used Internet transport protocols. TCP, which provides reliable data transmission, often introduces relatively large variations of the Internet delay due to its retransmission and congestion control mechanisms. Hence, UDP has been suggested as a transport protocol for Internetbased teleoperation systems even though it does not guarantee reliable data transmission and may cause data loss [1]. In this experiment, we employed a model for the Internet delay and used parameters typically observed in a UDP transmission [1], [7]. A random number generator was used to generate delay variations, which are shown in Figure 3. The maximum and average delays over a five-second interval were $132 \mathrm{msec}$ and $63 \mathrm{msec}$, respectively. In this experiment, we assumed that both the motion data transmitted to the slave controller and the force data fed to the master controller experienced the identical delay shown in Figure 3. 


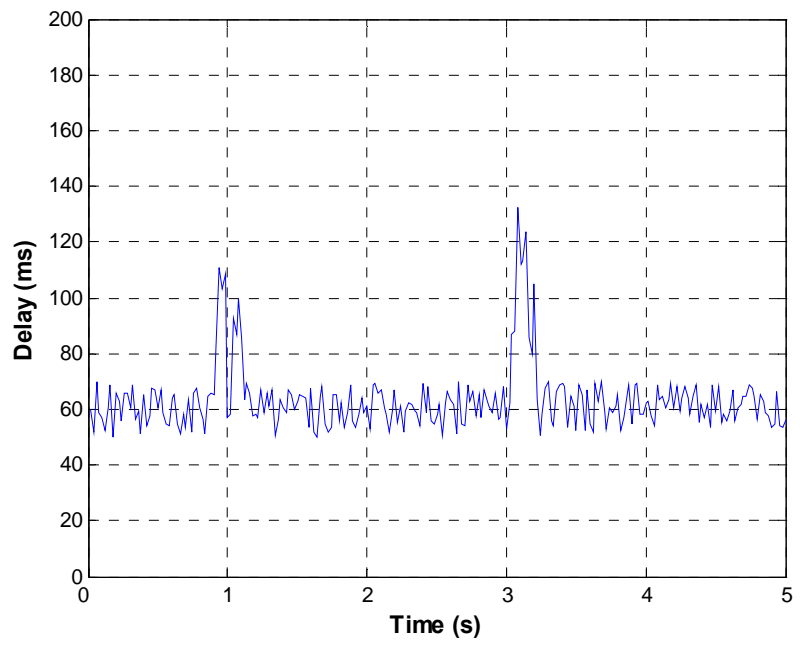

Figure 3. The Internet delay with UDP transmission.
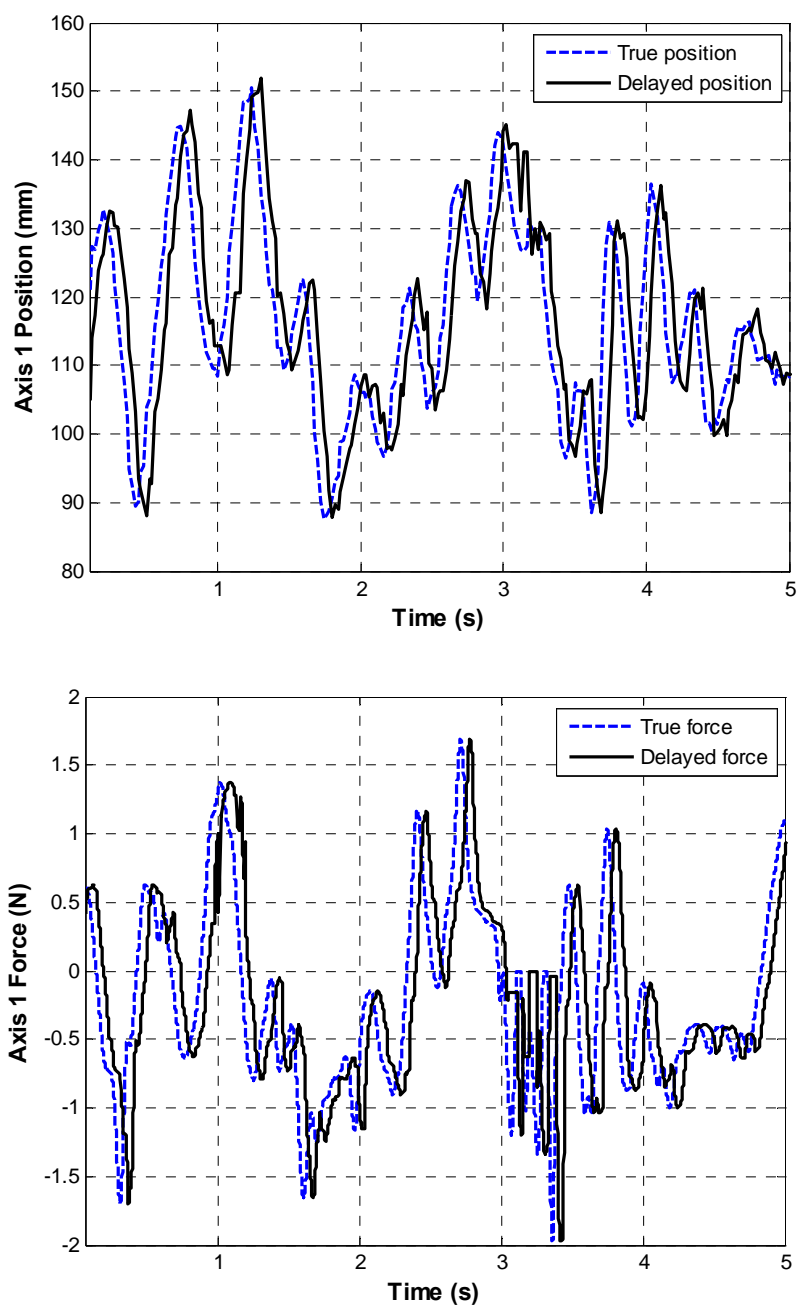

Figure 4. True and delayed motion data (top) and true and delayed feedback force data (bottom) collected from the master and slave controllers over a five-second interval.

\section{Motion and Force Prediction}

To verify the prediction performance of the particle filter method, one-dimensional motion and force data were collected over a five-second interval. Since the experimental scenario is based on the $3 \mathrm{D}$ virtual graphic representation, the sampling rate of the motion data was $50 \mathrm{~Hz}$ so that human eyes could perceive continuous motion. The sampling rate of the force data rendered by the PHANTOM Desktop haptic device was $1,000 \mathrm{~Hz}$ in order to maintain realistic force without discontinuity. In general haptic applications, it is advised that motion and force data should be sampled at no less than $30 \mathrm{~Hz}$ and $1,000 \mathrm{~Hz}$, respectively to prevent discontinuity of haptic data and to preserve closed-loops stability of an overall teleoperation system.
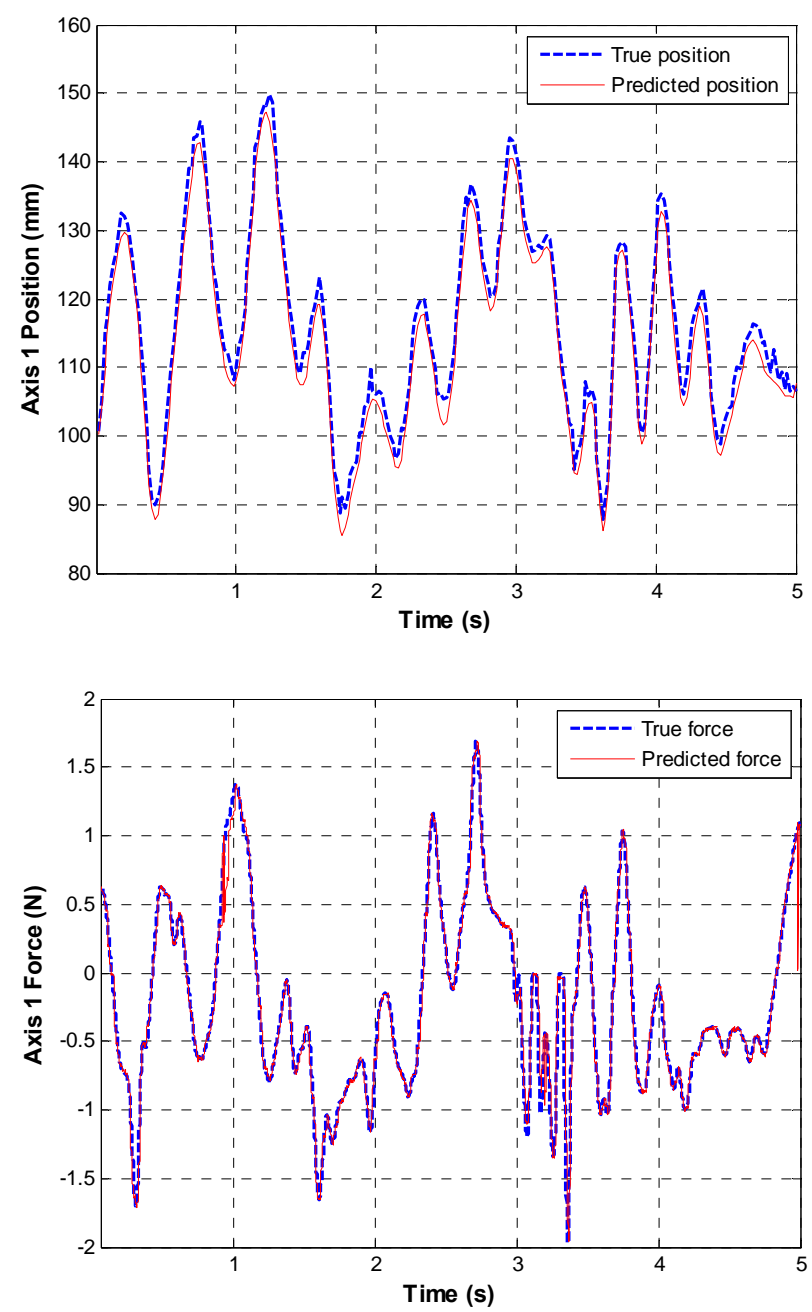

Figure 5. Predicted motion data (top) and feedback force data (bottom) collected from the slave and master controllers over a five-second interval. 
The one-dimensional motion and force data collected at the master controller and slave controller, respectively, are shown in Figure 4. Also shown are observations of the motion and force data that are delayed based on the delay shown in Figure 3. The delayed motion and force data are impaired by variations of the Internet delay as shown in Figure 4. Based on the collected motion and force data, we simulated non-Gaussian state noise using the Gamma density function (9) with $\alpha=2$ and $\beta=3$. We then evaluated the prediction performance of the particle filter method. In both motion and force prediction cases, we used 200 particles. The predicted motion and force data using the proposed particle filter method are shown in Figure 5. In order to compare them with the true data, transmission delays of the predicted motion and force data were not considered.

In general, a large number of particles improves the prediction performance. However, it introduces computational complexity that results in relatively longer computation time. In Internet-based teleoperation systems, such computational delay may affect stability of an overall teleoperation system. Hence, a number of particles should be selected efficiently to avoid an increase in the computational cost. In Table 2, the mean square errors (MSE) of the motion and force predictions are computed when the number of particles varies from 100 to 500. The MSE were measured when the Gamma density function was added as a non-Gaussian noise. Since errors tend to converge, a large number of particles may not be necessary.

Table 2. Number of Particles vs. MSE.

\begin{tabular}{ccc}
\hline \hline $\begin{array}{c}\text { Number of } \\
\text { particles }\end{array}$ & $\begin{array}{c}\text { Motion error } \\
(\mathrm{mm})\end{array}$ & $\begin{array}{c}\text { Force error } \\
(\mathrm{mN})\end{array}$ \\
\hline 100 & 2.824 & 192.5 \\
200 & 2.809 & 189.6 \\
300 & 2.724 & 187.1 \\
400 & 2.704 & 185.8 \\
500 & 2.680 & 179.7 \\
\hline \hline
\end{tabular}

* $\overline{\text { The motion and force units are millimeter and milliNewton, respectively. }}$.

\section{Comparison with the Kalman Filter}

The Kalman filter is a well-known recursive state predictor or estimator that provides an optimal solution in Bayesian probabilistic approaches. Since the traditional Kalman filter is only suitable in linear and Gaussian environments, the extended Kalman filter has been used to predict or estimate states of nonlinear dynamic systems [18]. In this experiment, we implemented the extended Kalman filter to the force prediction case shown in Figure 5. The comparison of the force prediction performance between the particle filter and extended Kalman filter is shown in Figure 6. Since the largest delay occurs between 3.0 and 3.5 seconds as shown in Figure 3, we present the comparison of prediction performance within that range in Figure 6. The MSE of the particle filter and extended Kalman filter are 198 and $846 \mathrm{mN}$, respectively. The proposed particle filter method outperforms the Kalman filter in nonlinear and non-Gaussian environments, as shown in Figure 6.

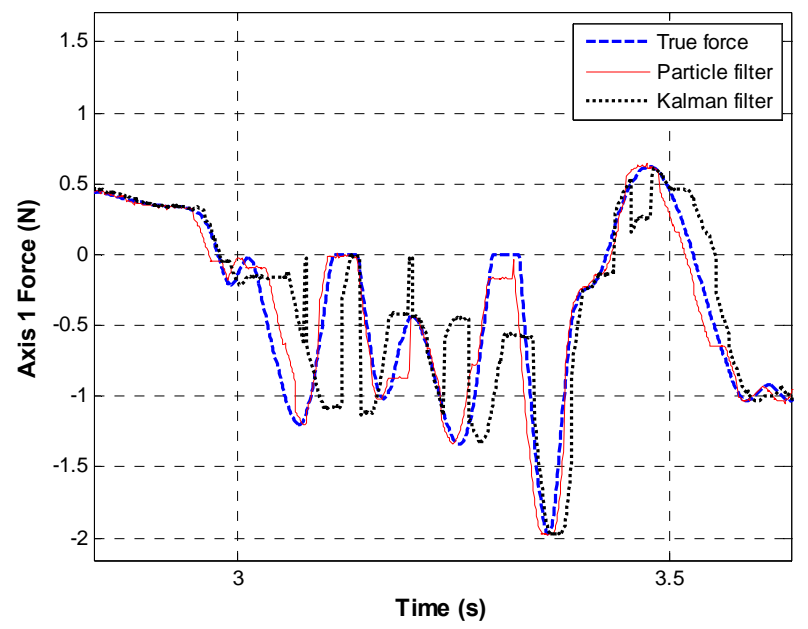

Figure 6. Prediction performance of particle filter and extended Kalman filter in nonlinear and non-Gaussian environments.

\section{DISCUSSION AND CONCLUSION}

In this paper, we presented motion and force predictions in Internet-based teleoperation systems. The prediction-based particle filter method was introduced and applied for motion and force data flows modeled by state-space formulations. Experiments using the haptic device in conjunction with a virtual teleoperator demonstrated that the particle filter method was well suited for predicting motion and force data, which may be impaired by the highly uncertain Internet delay. Furthermore, the particle filter method outperformed the conventional Kalman filter in extreme conditions such as nonlinear and non-Gaussian environments.

One limitation of the particle filter method is its computational complexity. Since this method is used in a closed-loop teleoperation system, computational complexity that may cause additional delay is undesirable. The particle filter method that adaptively selects a number of particles has been introduced for real-time tracking [19]. A large number of particles may be necessary in the case of predicting an initial state or highly uncertain state. Otherwise, a small number of particles may be used if uncertainty is low and motion and reflected force data at the next state are predictable.

In this paper, we introduced a signal processing approach to force-reflecting teleoperation systems to overcome the varying Internet delay. Such signal processing approach may be combined with an appropriate controller in order to address 
stability of an overall teleoperation system. In the case of constant delay, the wave variables transformation has been used to achieve stability [2]. However, in the presence of varying delay, it is difficult to address stability of an overall teleoperation system since motion and force data may be impaired during transmission. In this paper, we demonstrated that impairments of haptic data are compensated by a prediction-based approach. By introducing a novel controller, the proposed signal processing approach may enhance the stability even in the presence of the varying Internet delay.

\section{REFERENCES}

[1] E. Kamrani, H. Momeni, and A. Sharafat, "Modeling Internet delay dynamics for teleoperation," in Proc. IEEE Int. Conf. on Control Applications, Toronto, ON, Canada, Aug. 2005, pp. 1528-1533.

[2] G. Niemeyer and J. Slotine, "Designing force reflecting teleoperators with large time delays to appear as virtual tools," in Proc. IEEE Int. Conf. on Robotics and Automation, Albuquerque, NM, USA, Apr. 1997, pp. 2212-2218.

[3] K. Kawashima, K. Tadano, G, Sankaranarayana, and B. Hannaford, "Bilateral teleoperation with time delay using modified wave variables," in Proc. IEEE Int. Conf. on Intelligent Robots and Systems, Nice, France, Sept. 2008, pp. 424-429.

[4] T. Mirfakhrai and S. Payandeh, "On using delay prediction in controlling force reflecting teleoperation over the Internet," Robotica, vol. 23, no. 6, pp. 809-813, 2005.

[5] Y. Uchimura, T. Yakoh, and K. Ohnishi, "Bilateral robot system on the real-time network structure," IEEE Trans. on Industrial Electronics, vol. 51, no. 5, pp. 940-946, Oct. 2004.

[6] R. Wirz, M. Ferre, R. Marín, J. Barrio, J. Claver, and J. Ortego, "Efficient transport protocol for networked haptics applications," in Proc. The 6th Int. Conf. on Haptics: Perception, Devices, and Scenarios, Madrid, Spain, June 2008, vol. 5024, pp. 3-12.

[7] S. Clarke, G. Schillhuber, M. Zach, and H. Ulbrich, "Predictionbased methods for teleoperation across delayed networks," Springer-Verlag, Multimedia Systems, vol. 13, no. 4, pp. 253261, Oct. 2007.

[8] S. Clarke, G. Schillhuber, M. Zach, and H. Ulbrich, "The effects of simulated inertia and force prediction on delayed telepresence," Presence, vol. 16, no. 5, pp. 543-558, Oct. 2007.
[9] S. Munir and W. Book, "Internet-based teleoperation using wave variables with prediction," IEEE/ASME Trans. on Mechatronics, vol. 7, no 2, pp. 124-133, June 2002.

[10] M. Sanjeev Arulampalam, S. Maskell, N. Gordon, and T. Clapp, "A tutorial on particle filters for online nonlinear/non-Gaussian Bayesian tracking," IEEE Trans. on Signal Processing, vol. 50, no. 2, pp. 174-188, Feb. 2002.

[11] A. Doucet, N. de Freitas, and N. Gordon, "An introduction to sequential Monte Carlo methods," in Sequential Monte Carlo Methods in Practice, A. Doucet, N. de Freitas, and N. Gordon, Eds. New York: Springer-Varlag, 2001, pp. 4-14.

[12] R. Mottagi and S. Payandeh, "An overview of a probabilistic tracker for multiple cooperative tracking agents," IEEE Int. Conf. on Advanced Robotics, Seattle, WA, USA, July 2005, pp. 888-894.

[13] N. Fairfield, G. Kantor, and D. Wettergreen, "Towards particle filter SLAM with three dimensional evidence grids in a flooded subterranean environment," in Proc. IEEE Int. Conf. on Robotics and Automation, Orlando, FL, USA, May 2006, pp. 3575-3580.

[14] J. Lee, S. Payandeh, and L. Trajković, "Application of prediction-based particle filters for teleoperations over the Internet," in Proc. IASTED Int. Conf. on Robotics and Applications, Cambridge, MA, USA, Nov. 2009, pp. 22-27.

[15] G. Kitagawa and S. Sato, "Monte Carlo smoothing and selforganising state-space model," in Sequential Monte Carlo Methods in Practice, A. Doucet, N. de Freitas, and N. Gordon, Eds. New York: Springer-Varlag, 2001, pp. 177-195.

[16] F. Desbouvries and B. Ait-El-Fquih, "Direct versus predictionbased particle filter algorithm," in Proc. IEEE Workshop on Machine Learning for Signal Processing, Cancun, Mexico, Oct. 2008, pp. 303-308.

[17] L. Smith and V. Aitken, "The auxiliary extended and auxiliary unscented Kalman particle filters," in Proc. IEEE Canadian Conf. on Electrical and Computer Engineering, Vancouver, BC, Canada, Apr. 2007, pp. 1626-1630.

[18] T. Robertazzi and S. Schwartz, "On applying the extended Kalman filter to nonlinear regression models," IEEE Trans. on Aerospace and Electronic Systems, vol. 25, no. 3, pp. 433-438, May 1989.

[19] C. Kwok, D. Fox, and M. Meila, "Real-time particle filters," Proceedings of the IEEE, vol. 92, no. 2, pp. 469-484, Mar. 2004. 